\title{
Clinical Stabilization in Parkinson's Disease: The Multi-Target Treatment Description and Results
}

\section{Enrique de Font-Réaulx ${ }^{*}$}

Neurological Center, American British Cowdray Medical Center, Methodist International Network, Mexico, Email: defontreaulx@hotmail.com

${ }^{*}$ Corresponding authors: Enrique de Font-Réaulx, Neurological Center, American British Cowdray Medical Center, Methodist International Network, Mexico, E-mail: defontreaulx@hotmail.com

Received Date: June 24, 2016; Accepted Date: August 30, 2016; Published Date: September 02, 2016

Citation: Enrique de Font-Réaulx (2016) Clinical Stabilization in Parkinson's Disease: The Multi-Target Treatment Description and Results. J Neurophysiol Neurol Disord 3: 1-9.

\section{Abstract}

Previous clinical trials designed to control the progression of Parkinson's Disease (PD) have failed to demonstrate significant clinical stabilization. PD patients received a new oral Multi-Target Treatment to halt disease progression. The treatment consists of four substances that have a synergistic effect in controlling the most important known mechanisms of disease progression: aberrant apoptosis, oxidative damage, mitochondrial degeneration, caspase activation, syncytin-mediated neuroinflammation, and Mitogen-Activated Protein-Kinases activation.

Result: 40 patients with PD were studied, age 32 to 90 years (mean 62 years, SD +/-14.3), 23 female (57.5\%), 17 male (42.5\%). Initial United Parkinson's Disease Rating Scale Sub-section 3 (UPDRS-3) score: 1-15 (mean 5, SD +/- 2.5). Maximum follow-up period was 84 months, mean 38 months (SD +/-24.5).

Results: There were 2 clinical remissions (5\%), 34 patients (85\%) improved their basal UPDRS-3 score and 37 patients (92.5\%) had no increase in their UPDRS-3 score during the follow up period. The mean UPDRS-3 score at 51 months of follow-up was 3 . The UPDRS-3 was worse in only 3 patients $(7.5 \%)$.

Conclusion: At present, there is no medication that has proven effective in controlling PD's progression. The Multi-Target Therapy here described is a promising treatment that may control the progression of PD.

Keywords: Multi-Target therapy; Parkinson's Disease; Disease modifying treatment; Clinical stabilization; Apoptosis; Aeuroprotection; Neurodegeneration

\section{Background}

It is necessary to have a safe and effective treatment to control the progression of Parkinson's disease (PD). Currently, no such therapy exists. As this neurodegenerative disease progresses, the patient's quality of life and productivity drastically decrease. The cost of available treatment also increases exponentially as the disease progresses. At the earlier stages, the annual cost is from $\$ 2,500$ to $\$ 7,000$ on medication alone; while long-term care costs about $\$ 47,000$ per year [1].

(C)2016 The Authors. Published by the JScholar under the terms of the Creative Commons Attribution License http://creativecommons.org/licenses/ by/3.0/, which permits unrestricted use, provided the original author and source are credited.

\begin{abstract}
Previous clinical trials designed to control Parkinson's disease progression

Currently, the medical options available for PD are mainly for symptomatic treatment. The first clinical trial designed to evaluate a medication for disease-modifying potential was the Deprenyl and Tocopherol Antioxidative Therapy for Parkinson's Disease (DATATOP study) [2].

Patients with PD at an early stage were randomly assigned to take placebo or selegiline, the medication of interest, and the primary outcome was disease progression to disability such that the patient required levodopa therapy.
\end{abstract}


This study reported that patients who were treated with selegiline had about a $50 \%$ lower risk of reaching that endpoint, but this was likely due to symptomatic improvement rather than modification of disease progression.

The Sinemet vs Deprenyl in PD (SIN-DEP-PAR) [3] was the first study that used a wash out period after treatment with selegiline to try to differentiate between symptomatic improvement and true disease modification. Patients with very early PD were randomly assigned to treatment with selegiline or placebo, and to symptomatic treatment with bromocriptine or carbidopa/levodopa. Over 14 months of follow-up, selegiline was stopped 2 months prior to endpoint, and the bromocriptine and levodopa were stopped 1 week prior to endpoint. Patients treated with selegiline were clinically better at 14 months compared to those who received placebo, potentially consistent with a slowing of disease progression. However, monoamine oxidase- $\mathrm{B}$ (MAO-B) inhibitors like selegiline bind irreversibly to $\mathrm{MAO}-\mathrm{B}$ in the brain and it takes the brain about 30 days to turn over half of its MAO-B; thus, there could have been a remnant symptomatic effect that was not sufficiently washed out by the study endpoint.

The Earlier vs Later Levodopa Therapy in Parkinson Disease (ELLDOPA) study [4] compared levodopa at various dosages to placebo for 40 weeks, followed by a 2 -week washout period. After 40 weeks, symptom improvement was positively correlated with the dose of levodopa. After the 2-week washout, benefits of levodopa declined but treated patients still had fewer symptoms than those who received placebo. Thus, it was suspected that this treatment could provide disease modification. However, 2 weeks may not be enough time to eliminate the symptomatic effects of levodopa, which is hypothesized to induce compensatory changes to dopamine neurons, with an unknown duration of lasting effect. There were 2 delayed-start studies for rasagiline: TEMPO [TVP-1012 in Early Monotherapy for Parkinson's Disease Outpatients] [5,6] and ADAGIO [Attenuation of Disease progression with Azilect Given Once daily [7].

In the TEMPO study [5] about 400 patients were randomly assigned to 1 of 3 groups: rasagiline $1 \mathrm{mg}$ /day for 12 months, $2 \mathrm{mg} /$ day for 12 months, or placebo for 6 months followed by rasagiline $2 \mathrm{mg}$ /day for the second 6 months. The latter group is the delayed-start group. At the end of 12 months, a small but statistically significant difference was found, with the early-start groups clinically better than the delayed-start group.

The ADAGIO study [7] included approximately 1200 patients with a total follow-up of 18 months. It compared rasagiline 1 mg early start vs $1 \mathrm{mg}$ delayed start, and $2 \mathrm{mg}$ early start vs 2 mg delayed start. The delayed-start groups received placebo for 9 months followed by active medication for 9 months; the early-start groups received rasagiline for the full period of 18 months. Patients who received rasagiline $1 \mathrm{mg}$ early did better than the $1 \mathrm{mg}$ delayed group.
It was a small but statistically significant difference at 18 months, findings consistent with the TEMPO results. In the $2 \mathrm{mg}$ group there was no difference between the early and delayed start groups.

Thus far, no symptomatic medication has proven to be neuroprotective or have a clear disease modifying effect. There is also no reason to support that they might achieve this goal based on their mechanisms of action as none of them has effect in syncytin-mediated neuroinflammation, free radical control, caspase inhibition, activation of Mitogen-Activated ProteinKinases (MAPK), or other mechanism of PD progression.

As PD's progression is a very complex process, with several simultaneous mechanisms of action, we postulate that it is necessary to combine more than one drug in order to effectively control the disease progression. We describe a new oral treatment, called Multi-Target Therapy (MTT), specifically designed to control neurodegenerative disease progression and to obtain clinical stabilization. Each one of its components has a different pathophysiologic target, and no symptomatic effect, different from what has been studied previously.

The pathological principle of protein propagation offers new disease-modifying therapeutic approaches to treating neurodegenerative diseases. It is well known, that in prion diseases, an infectious protein replicates by recruiting and inducing pathological conformational changes in its normal counterpart, resulting in the aggregation of pathological prions [8-10]. Prions thus act as corruptive templates that induce a chainreaction-like process of protein misfolding and progressive aggregation. Recent studies have provided convincing evidence that a 'prion-like' self-propagating mechanism may apply to a wider range of proteins that are associated with neurodegenerative diseases, including misfolded $A \beta$, tau and $\alpha$-synuclein, mutant huntingtin with polyglutamine repeats (characteristic of Huntington disease), mutant superoxide dismutase 1 (SOD1) and phosphorylated TDP43 [11].

Studies in vivo on individuals with PD who had received transplants of fetal mesencephalic dopaminergic neurons, show that $\alpha$-synuclein pathology can propagate, as they developed a-synuclein-positive Lewy bodies and showed signs of neuronal degeneration [12-16]. Evidence from in vitro studies [17-19] and animal model experiments [17,18,20-27] confirmed seeded aggregation and transmission of a-synuclein [11].

Fluid-phase and receptor-mediated endocytosis have been implicated in the cellular uptake of mutant tau, mutant SOD1, and fibrils, oligomers and monomers of a-synuclein [19,2832 ]. It is possible that these proteins could have synergistic deleterious effects on mitochondrial function [33].

The earliest lesions in PD and in dementia with Lewy bodies can be detected in the olfactory bulb and anterior olfactory nucleus, as well as in the dorsal motor nucleus of the vagus nerve in the medulla oblongata [11]. 
In PD and in dementia with Lewy bodies, with increasing burden of pathology, $\alpha$-synuclein aggregate pathology is found in the pons and midbrain before being found in the basal forebrain and, ultimately, in the neocortex [11,34-39]. Thus, only in more advanced stages of PD does $\alpha$-synuclein aggregation cause the loss of midbrain dopaminergic neurons in the pars compacta of the substantia nigra $[11,34]$. The accumulation of $\alpha$-synuclein aggregates in the anterior olfactory nucleus $[11,40]$ and olfactory bulb is clinically reflected by hyposmia, which is frequently observed before the onset of motor symptoms in PD [11,41]. It has been suggested that projection neurons with sparsely myelinated axons would require prodigious energy expenditure to maintain axonal function and transport [42], and that such high energy demands would result in continuously high levels of oxidative stress that could increase neuron vulnerability to $\alpha$-synuclein aggregation in PD [11,43-46].

Template-directed replication and subsequent cell-cell transmission of pathology-associated proteins provides a common molecular pathway that could be targeted by novel therapeutic strategies with the aim of disrupting or delaying propagation. Moreover, agents that interfere with the release or uptake of neurodegenerative disease proteins could prevent transmission of pathology to neighboring neurons. Finally, many neurodegenerative diseases are likely to be non-cell autonomous, with an important part played by astroglia, oligodendroglia and microglia [11].

While designing the MTT we considered syncytin-mediated neuroinflammation a therapeutically important target. Even though increased Human Endogenous Retro-Virus (HERV) activity could be an epiphenomena, and association does not imply causality, there are increasing and stronger evidence that the presence of HERV-induced syncytin production is correlated to higher progression index in most of the neurodegenerative diseases studied. It also has a well-studied neuroinflammatory pathway, where the released tumor necrosis factor alpha (TNF $\alpha$ ) bind to the Tumor Necrosis Factor Receptor (TNFR) that initiates necrosis factor $\kappa \beta$ (NF- $\kappa \beta$ ) activation in an autocrine and paracrine manner. The signaling pathway downstream from TNFR leads to nuclear translocation of NF$\kappa \beta$, where it binds to the promoters of syncytin1. Cytoplasmic accumulation of env proteins induces endoplasmic reticulum (ER) stress responses and extracellular or transmembrane env proteins can exert their properties: fusogenicity and capacity to activate the neuroimmune system, damage oligodendrocytes and interfere with myelin regeneration [47]. Additionally, the long terminal repeats (LTRs), which control retroviral gene expression, can also change host RNA levels [48] and the HERV-expression pattern has been shown to be altered in murine cell culture models for prion infection [49] and in the cerebrospinal fluid (CSF) of sporadic CJD patients [50,51].

\section{The multi-target therapy to control neurodegen-} erative diseases' progression

The Multi-Target Therapy treatment (patent MX329006 B), combines four substances that interact to produce a synergistic effect. As far as we know, this is the first treatment specifically designed to control the progression of neurodegenerative diseases such as PD. It contains ferulic acid $50 \mathrm{mg}$, apigenin 100 $\mathrm{mg}$, gamma oryzanol (GO) $50 \mathrm{mg}$ and sylimarin $150 \mathrm{mg}$. It is designed to preserve the brain's physiological micro-environment and to control the most active known pathophysiologic mechanisms involved in neurodegenerative disease progression. Because of the compound's antioxidant properties, it has a protective effect for myelin and neurons; thus it may also be useful in other neurodegenerative oxidative-mediated diseases, such as multiple sclerosis and Alzheimer's disease, among others. The MTT is an oral treatment designed to be taken daily and long-term to continuously combat the active pathophysiologic mechanisms involved in disease progression. It is not designed to relieve the symptoms of PD, but to slow down and control the progression of the disease. All the components have been tested previously in humans and proven to be safe for consumption [52].

\section{Ferulic acid}

Ferulic Acid (FA) has shown protective effects against various inflammatory diseases [53]. It is an antioxidant that has proved highly effective in neutralizing free radicals such as superoxide, hydroxyl radical, and nitric oxide (NO). It acts synergistically with other antioxidants, giving them extra potency [54], and protects against nitrosamines [55]. Its structure is similar to catecholamines like norepinephrine. There are no documented side effects of ingestion of FA in humans. FA is a fenolcarboxilic acid [56] that has been shown in vitro and in vivo to decrease death of oligodendrocytes under cellular stress, which can improve neurological outcome [57]. Several mechanisms of disease can cause damage in the central nervous system that can modify the core expression of glial cells. Those effects can be produced by protein misfolding or by protein accumulation in the ER, which causes a cellular stress response and the production of neurotoxic molecules, including redox reactants like NO, reactive nitrogen-oxygen species, peroxinitrite anions and superoxide, which can cause encephalic damage [57]. FA acts against several potent cellular stress mechanisms mediated by Interleukin 1 (IL1), inducible nitric oxide synthase (iNOS) and redox reactant synthesis, among others.

Syncytin is a 518-amino-acid membrane glycoprotein that may exert biological action by binding to the receptor ASCT2 (alanine, serine, cysteine transporter 2), which is both an amino acid transporter and a retrovirus receptor [58]. Viral envelope glycoproteins are known to affect immune responses and syncytin is related to activation of lymphocytes and macrophages [59]. Overexpression of syncytin in astrocytes and macrophages is sufficient to cause the cells to produce high amounts of the proinflammatory cytokine IL-1 $\beta$ and reactive oxygen radicals. The overexpression of syncytin is toxic to oligodendrocytes, and this toxicity is prevented by FA [60]. 
FA also appears to encourage the proliferation of at least some types of nerve cells, such as retinal cells [61]. It has proven to be effective in several diseases in humans and in animals. In a model of iron-induced neuronal oxidative stress and neuronal apoptosis in granular cerebellar cells [62], an increase in caspase 3 activity and apoptosis related to gene p53 activity and of its gene effector p21 was demonstrated. In neurons treated with tetrametilpirazine and FA and then exposed to the ironinduced oxidative model, a significant decrease of caspase 3 activity and expression of p53 and p21 was documented, with less severe oxidative damage and apoptosis induced by iron. This suggests that tetrametilpirazine and FA can be used to treat neurological diseases associated to oxidative stress.

Macrophage activity also has been related to neurodegenerative disease progression. FA has been shown to specifically reduce the macrophage inflammatory protein-2 (MIP-2) in the RAW264.7 macrophage cell line. Its effect was superior to that of dexamethasone [63]. FA-related compounds have potential to produce NSAID-like effects $[64,65]$, can inhibit NO production [66] and have radical scavenging activity [67].

\section{Apigenin}

Apigenin is a flavonoid found in its natural form in several fruits and vegetables. It is well known for its anti-oncogenic, antioxidant and anxiolytic properties, acting by several mechanisms. In cases of injury or disease, microglia are recruited to the site of damage and become ativated, as evidenced by morphological changes and expression of pro-inflammatory cytokines. Evidence suggests that microglia proliferate by cell division to create gliosis at the site of injury, such as the amyloid plaques in Alzheimer's disease and the substantia nigra in PD. The hyperactivation of microglia contributes to neurotoxicity. Anti-inflammatory compounds modulate the progression of the cell cycle and induce apoptosis of activated cells, and thus might inhibit microglial proliferation [68].

Apigenin, among its structural analogues, appears to be the most potent inhibitor of the production of pro-inflammatory cytokines by lipopolysaccharide-stimulated human peripheral blood mononuclear cells [69]. Apigenin inhibits phosphorylation pathways and is a potential inhibitor of cellular autoimmunity. Due to the inhibitory activity of flavonoids on IL-4 and IL-13 synthesis, it can be expected that the intake of flavonoids, depending on the quantity and quality, may ameliorate allergic symptoms or prevent the onset of allergic diseases [70]. Apigenin is a very potent inhibitor of xanthine oxidase activity. Prostaglandin biosynthesis and NO production have been implicated in the processes of neurodegeneration, carcinogenesis and inflammation. Apigenin may be the most potent inhibitor of transcriptional activation of both COX2 and iNOS. Western and northern blot analyses demonstrated that apigenin significantly blocked protein and mRNA expression of COX2 and iNOS in LPS-activated macrophages. Transient transfection experiments showed that LPS caused an approximately 4-fold increase in both COX2 and iNOS promoter activities, but these elevations were suppressed by apigenin. This suggests that modulation of COX 2 and iNOS by apigenin may be important in the prevention of carcinogenesis and inflammation [71].
The chemical chain reaction initiated by NMDA receptors and by calcium influx causes an increase in the activity of the enzyme system known as the Mitogen-Activated Protein Kinases system (MAPK). Factors released in response to hypoxia (growth factors, inflammatory cytokines and free radicals) can also stimulate the MAPK system. MAPK activity can remain elevated long after cessation of the initial stimulus. Inhibiting MAPK activity has been shown to have a neuroprotective effect in cases of central nervous system insult; and apigenin has been shown to be a strong MAPK inhibitor [72].

Decreased activity of superoxide dismutase was strongly correlated with increased oxidative damage to plasma proteins at the individual level. Intervention with apigenin seemed to partly overcome this decrease and resulted in increased levels of glutathione reductase and superoxide dismutase [73].

\section{Sylimarine}

Sylimarine is an antioxidant found in several plants and types of food. It is estimated that its antioxidant effect is 10 times stronger than that of vitamin E. It increases the hepatic content of the antioxidant enzyme glutathione by $35 \%$ and decreases free radical-mediated cellular damage by inhibition of lipoxygenase. Lipoxygenase acts in polyunsaturated fatty acids and produces leukotrienes, which are involved in cellular membrane damage [74].

\section{Gamma oryzanol}

GO was discovered in the late 60 s while experimenting with growth factors in animals. It was observed that GO accelerated animal growth with no collateral effects. It is a compound molecule made up of FA and estherol. When GO is taken orally, absorption is low $(<10 \%)$. Once absorbed by the digestive system, it is hydrolyzed by a non-specific esterase, separating it into free FA and estherol. When administered alone, FA absorption increases by a factor of 20 to 30 because it is a hydrosoluble compound, thus eliminating the issue of cellular transport. Also, free FA does not undergo first pass metabolism by the liver as GO does. Nevertheless, GO is an additional source of FA that increases the level of this antioxidant, providing additional effect [75].

\section{Clinical stabilization treatment trial}

The 4 different substances of the MTT, synergistically working against the most important known pathophysiologic mechanisms of disease progression, including pathophysiologic oxidation, mitochondrial degeneration, cellular damage mediated by macrophage free radicals, caspase activation, aberrant apoptosis, and MAPK cellular damage, could preserve the physical and functional integrity of the myelin-axon unit, the neurovascular unit, and the neurons and glial cells, achieving clinical stability in neurodegenerative disease. We have previously demonstrated that it is safe to use the MTT in humans [52]. It is the first, and as far as we know, the only therapy that combats the most known mechanisms of disease progression simultaneously. As the MTT has no effect on the clinical symptoms of $\mathrm{PD}$, it does not replace standard symptomatic treatment. 


\section{Materials and Methods}

We designed a cohort phase II/III trial. The primary endpoint was the United Parkinson's Disease Rating Scale-3 (UPDRS-3) score during the follow up period (a lower score indicates better clinical condition). Before beginning the trial, we optimized the symptoms of each participant and recorded their basal UPDRS-3 score. Then, we added the MTT at a dose of 1 pill every 12 hours taken on an empty stomach. We evaluated all patients with clinical examination, UPDRS-3 scoring and laboratory testing every 3 months during the follow-up period of up to 84 months.

\section{Results}

We included 40 patients with PD. Age: 32 to 90 years (mean 62 years, SD +/- 14.3), 23 female (57.5\%), 17 male (42.5\%). Initial UPDRS-3 score: 1-15 (mean 5, SD +/- 2.5). Maximum followup period: 84 months, mean 38 months (SD +/- 24.5). During the follow up period, 7 patients $(17.5 \%)$ improved their basal UPDRS-3 score while there was no change in score for 37 patients $(92.5 \%)$. There were 2 clinical remissions (5\%). The UPDRS-3 was worse in 3 patients $(7.5 \%)$. Of these three patients, one discontinued the MTT for 18 months and deteriorated in that time. The mean UPDRS-3 score was 3 at 51 months of follow-up. Adverse effects: None.

\section{Conclusions}

One of the most important challenges in PD is controlling disease progression. The PD progression index has been established in a deterioration of $7.8+/-9.0$ points in UPDRS at 42 weeks of follow-up [76] in the UPDRS. It is necessary to have new therapeutic resources to control disease progression and improve patient outcomes. As there is no medication that has previously demonstrated efficacy in controlling PD progression, it is justified to design and test new medications created for this purpose.

The MTT is the first treatment specifically designed to control the progression of neurodegenerative diseases. With this initial evidence, we believe that the MTT may be able to change the course of PD and control its progression. The MTT represents a new pharmacological strategy in PD treatment because it combines several agents with different mechanisms of action, which has never been done before in attempting to halt neurodegenerative disease, and particularly $\mathrm{PD}$, progression.

\section{FOLLOW UP IN PD PATIENTS TREATED WITH THE MTT}

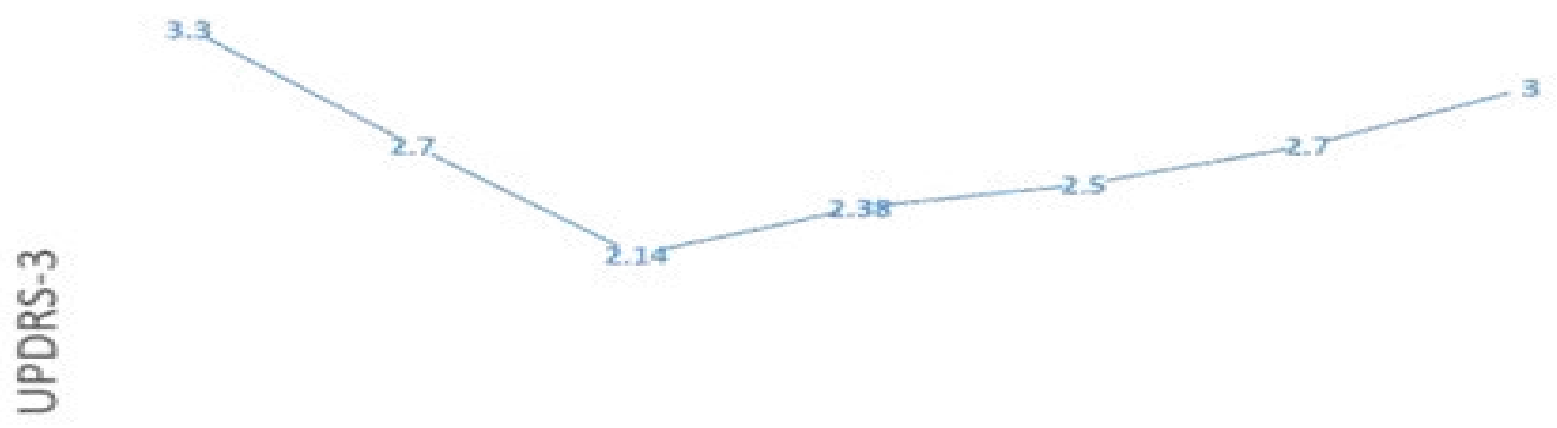

12 monhs 24 manths 36 months 48 months 60 months 72 moethi 84 months

Figure 1: Disease progression control in PD patients using the Multi-Target Therapy. Historically, the score of PD patients treated only for symptomatic improvement would increase as time went on, affecting their quality of life. In PD patients treated with the Multi-Target Therapy, the disease has less clinical progression in most of the cases during the follow up period. 


\section{References}

1) Huse DM, Schulman K, Orsini L, et al. (2005) Burden of illness in Parkinson's disease. Mov Disord 20: 1449-1454.

2) The Parkinson Study Group (1989) Effect of deprenyl on the progression of disability in early Parkinson's disease. N Engl J Med 321: 13 64-1371.

3) Olanow CW, Hauser RA, Gauger L, et al. (1995) The effect of deprenyl and levodopa on the progression of Parkinson's disease. Ann Neurol. 38: 771-777.

4) Fahn S, Oakes D, Shoulson I, Kieburtz K, Rudolph A, Lang A, Olanow CW, et al. (2004) The Parkinson Study Group. Levodopa and the progression of Parkinson's disease. N Engl J Med 351: 2498-2508.

5) The Parkinson Study Group (2002) Dopamine transporter brain imaging to assess the effects of pramipexole vs levodopa on Parkinson disease progression. JAMA 287: 1653-1661.

6) Whone AL, Watts RL, Stoessl AJ, et al. (2003) Slower progression of Parkinson's disease with ropinirole versus levodopa: the REAL-PET study. Ann Neurol 54: 93-101.

7) The Parkinson Study Group (2004) A controlled, randomized, delayed-start study of rasagiline in early Pakinson disease. Arch Neurol 61: 561-566.

8) Prusiner SB (1982) Novel proteinaceous infectious particles cause scrapie. Science. 216:136-144.

9) Aguzzi A, Sigurdson C, Heikenwaelder M (2008) Molecular mechanisms of prion pathogenesis. Annu. Rev. Pathol 3:11-40.

10) Aguzzi A (2009) Cell biology: beyond the prion principle. Nature 459: 924-925.

11) Brettschneider J, Del Tredici K, Lee VM-Y Trojanowski JQ (2015) Spreading of pathology in neurodegenerative diseases: a focus on human studies. Nat Rev Neurosci 16: 109-120.

12) Li JY, Englund E, Holton JL, Soulet D, Hagell P, et al. (2008) Lewy bodies in grafted neurons in subjects with Parkinson's disease suggest host-to-graft disease propagation. Nature Med. 14:501-503.

13) Kordower JH, Chu Y, Hauser RA, Olanow CW, Freeman TB (2008) Transplanted dopaminergic neurons develop PD pathologic changes: a second case report. Mov. Disord 23: 2303-2306.

14) Li JY, Englund E, Widner H, Rehncrona S, Björklund A, Lindvall O, et al. (2010) Characterization of Lewy body pathology in 12- and 16 -year-old intrastriatal mesencephalic grafts surviving in a patient with Parkinson's disease. Mov. Disord 25: 1091-1096.

15) Chu Y, Kordower JH (2010) Lewy body pathology in fetal grafts. Ann. NY Acad. Sci 1184: 55-67.

16) Kurowska Z, et al. (2011) Signs of degeneration in 12-22-year old grafts of mesencephalic dopamine neurons in patients with Parkinson's disease. J. Parkinsons Dis 1: 83-92.

17) Hansen C, et al. (2011) $\alpha$-synuclein propagates from mouse brain to grafted dopaminergic neurons and seeds aggregation in cultured human cells. J. Clin. Invest 121: 715-725.

18) Kordower JH, et al. (2011) Transfer of host-derived a synuclein to grafted dopaminergic neurons in rat. Neurobiol. Dis 43: 552-557.

19) Volpicelli-Daley LA, et al. (2011) Exogenous $\alpha$-synuclein fibrils induce Lewy body pathology leading to synaptic dysfunction and neuron death. Neuron 72: 57-71.

20) Desplats $P$, et al. (2009) Inclusion formation and neuronal cell death through neuron-to-neuron transmission of alpha-synuclein.. Proc Natl Acad Sci U S A. 106: 13010-13015.

21) Mougenot AL, Nicot S, Bencsik A, Morignat E, et al. (2012) Prion-like acceleration of a synucleinopathy in a transgenic mouse model. Neurobiol Aging 33: 2225-2228.

22) Luk KC, Kehm VM, Zhang B, O'Brien P, et al. (2012) Intracerebral inoculation of pathological $\alpha$-synuclein initiates a rapidly progressive neurodegenerative $\alpha$-synucleinopathy in mice. J. Exp. Med 209: 975986.
23) Masuda-Suzukake M, Nonaka T, Hosokawa M, Oikawa T, et al. (2013) Prion-like spreading of pathological $\alpha$-synuclein in brain. Brain 136: 1128-1138.

24) Luk KC, et al. (2012) Pathological $\alpha$-synuclein transmission initiates Parkinson-like neurodegeneration in nontransgenic mice. Science 338: 949-953.

25) Watts JC, Giles K, Oehler A et al. (2013) Transmission of multiple system atrophy prions to transgenic mice. Proc. Natl Acad. Sci. USA 110: 19555-19560.

26) Recasens A, et al. (2014) Lewy body extracts from Parkinson disease brains trigger $\alpha$-synuclein pathology and neurodegeneration in mice and monkeys. Ann. Neurol 75: 351-362.

27) Angot E, et al. (2012) Alpha-synuclein cell-to-cell transfer and seeding in grafted dopaminergic neurons in vivo. PLoS One 7: e39465. 28) Frost B, Jacks RL, Diamond MI (2009) Propagation of tau misfolding from the outside to the inside of a cell. J. Biol Chem 284: 12845-12852.

29) Munch C, O’Brien J, Bertolotti A (2011) Prion-like propagation of mutant superoxide dismutase-1 misfolding in neuronal cells. Proc. Natl Acad. Sci. USA 108: 3548-3553.

30) Holmes BB, et al. (2013) Heparan sulfate proteoglycans mediate internalization and propagation of specific proteopathic seeds. Proc. Natl Acad. Sci. USA 110: E3138-E3147.

31) Wu JW, et al. (2013) Small misfolded Tau species are internalized via bulk endocytosis and anterogradely and retrogradely transported in neurons. J Biol Chem 288: 1856-1870.

32) Lee HJ, et al. (2008) Assembly-dependent endocytosis and clearance of extracellular alpha-synuclein. Int. J. Biochem. Cell Biol 40: 1835-1849.

33) Rhein V, et al (2009) Amyloid-beta and tau synergistically impair the oxidative phosphorylation system in triple transgenic Alzheimer's disease mice. Proc. Natl Acad. Sci. USA. 106: 20057-20062.

34) Braak H, et al. (2003) Staging of brain pathology related to sporadic Parkinson's disease. Neurobiol. Aging. 24: 197-211.

35) Del Tredici K, Rub U, De Vos RA, Bohl JR, Braak H (2002) Where does parkinson disease pathology begin in the brain? J. Neuropathol. Exp. Neurol 61: 413-426.

36) Del Tredici K, Braak H (2012) Spinal cord lesions in sporadic Parkinson's disease. Acta Neuropathol. (Berl.) 124: 643-664.

37) Braak H, Del Tredici K. (2009) Neuroanatomy and pathology of sporadic Parkinson's disease. Adv. Anat. Embryol. Cell Biol 201: 1-119.

38) van de Berg WD, et al. (2012) Patterns of $\alpha$-synuclein pathology in incidental cases and clinical subtypes of Parkinson's disease. Parkinsonism Relat. Disord. 18: S28-S30.

39) Goedert M, Spillantini MG, Del Tredici K, Braak H (2013) 100 years of Lewy pathology. Nature Rev. Neurol 9: 13-24.

40) Pearce RK, Hawkes CH, Daniel SE (1995) The anterior olfactory nucleus in Parkinson's disease. Mov. Disord 10: 283-287.

41) Hawkes CH, Shephard BC, Daniel SE (1999) Is Parkinson's disease a primary olfactory disorder? QJM 92: 473-480.

42) Aiello GL, Bach-y-Rita P (2000) The cost of an action potential. J. Neurosci. Methods 103: 145-149.

43) Giasson BI, et al. (2000) Oxidative damage linked to neurodegeneration by selective $\alpha$-synuclein nitration in synucleinopathy lesions. Science 290: 985-989.

44) Chung CY, et al. (2013) Identification and rescue of $\alpha$-synuclein toxicity in Parkinson patient-derived neurons. Science. 342: 983-987. 
45) Ryan SD, et al. (2013) Isogenic human iPSC Parkinson's model shows nitrosative stress-induced dysfunction in MEF2-PGC1a transcription. Cell 155: 1351-1364.

46) Zuo L, Motherwell MS (2013) The impact of reactive oxygen species and genetic mitochondrial mutations in Parkinson's disease. Gene 532: 18-23.

47) Dolei A, Uleri E, Ibba G, Caocci M, Piu C, et al. (2015) The aliens inside human DNA: HERV-W/MSRV/syncytin-1 endogenous retroviruses and neurodegeneration. J Infect Dev Ctries 9: 577-587.

48) Denner J. Endogenous Retroviruses. In Retroviruses: Kurth R, Bannert N. Hethersett (eds) Molecular Biology, Genomics and Pathogenesis. Norwich, UK: Caister Academic Press.

49) Stengel A, Bach C, Vorberg I, Frank O, Gilch S, et al. (2006) Prion infection influences murine endogenous retrovirus expression in neuronal cells. Biochemical and biophysical research communications 343: 825-831.

50) Jeong BH, Lee YJ, Carp RI, Kim YS (2010) The prevalence of human endogenous retroviruses in cerebrospinal fluids from patients with sporadic Creutzfeldt-Jakob disease. J Clin Virol 47: 136-142.

51) Greenwood AD, Vincendeau M, Schmädicke AC, Montag J, Seifarth W, Motzkus D. (2011) Bovine spongiform encephalopathy infection alters endogenous retrovirus expression in distinct brain regions of cynomolgus macaques (Macaca fascicularis). Molecular Neurodegeneration. 6: 44.

52) de Font-Réaulx E, Dorazco-Barragán G (2010) [Clinical stabilisation in neurodegenerative diseases: clinical study in phase II]. Rev Neurol 50: 520-528.

53) Graf E (1992) Antioxidant potential of ferulic acid. Free Radical Biology and Medicine. 13: 435-448.

54) Trombino S, Serini S, Di Nicuolo F, Celleno L, et.al. (2004) Antioxidant effect of ferulic acid in isolated membranes and intact cells: synergistic interactions with alpha-tocopherol, beta-carotene, and ascorbic acid. J Agric Food Chem 52: 2411-2420.

55) Kuenzig W, Chau J, Norkus E, Holowaschenko H, Newmark H, Mergens W, Conney AH (1984) Caffeic and ferulic acid as blockers of nitrosamine formation. Carcinogenesis 5: 309-313.

56) Ogiwara T, Satoh K; Negoro T; Okayasu H; Sakagami H; Fujisawa S (2003) Inhibition of NO production by activated macrophages by phenol-carboxylic acid monomers and polymers with radical scavenging activity. Anticancer Res. 23: 1371-1323.

57) Antony JM, van Marle G, Opii W, Butterfield DA, Mallet F, Wee Yong V, et al. (2004) Human endogenous retrovirus glycoprotein-mediated induction of redox reactants causes oligodendrocyte death and demyelination. Nature Neuroscience 7: 1088-1095.

58) Kudo Y, Boyd CA, Sargent IL, Redman CW (2003) Hypoxia alters expression and function of syncytin and its receptor during trophoblast cell fusion of human placental BeWo cells: implications for impaired trophoblast syncytialisation in pre-eclampsia. Biochim Biophys Acta 1638: 63-71.

59) Mi S, Lee X, Li X, Veldeman GM, Finnerty H, et al.(2000) Syncytin is a captive retroviral envelope protein involved in human placental morphogenesis. Nature 403: 785-789.

60) Mattson MP, Taub DD. (2004) Ancient viral protein enrages astrocytes in multiple sclerosis. Nat Neurosci 7: 1021-123.

61) Sohn YT, Oh JH. (2003) Characterization of physicochemical properties of ferulic acid. Arch Pharm Res 26: 1002-1008.

62) Zhaohui Zhang, Taotao Wei, Jingwu Hou, Gengshan Li, Shaozu Yu, Wenjuan Xin (2003) Iron-induced oxidative damage and apoptosis in cerebellar granule cells: attenuation by tetramethylpyrazine and ferulic acid. European Journal of Pharmacology 467: 41-47.
63) Sakai S. Ochiai H.; Nakajima K. Terasawa K (1997) Inhibitory effect of ferulic acid on macrophague inflammatory protein-2 production in a murine macrophague cell line, RAW264.7. Cytokine 9: 242-248.

64) Ogiwara T, Satoh K, Kadoma $Y$, et al. Radical scavenging activity and cytotoxicity of ferulic acid. Anticancer Res 22: 2711-2717.

65) Hirata A, Murakami Y, Atsumi T, Shoji M, et al. (2005) Ferulic acid dimer inhibits lipopolysaccharide-stimulated cyclooxygenase-2 expression in macrophages. In Vivo 19: 849-53.

66) Ogiwara T, Satoh K, Negoro T, Okayasu H, Sakagami H, Fujisawa S (2003) Inhibition of NO production by activated macrophages by phenolcarboxylic acid monomers and polymers with radical scavenging activity. Anticancer Res 23: 1317-1323.

67) Ogiwara T, Satoh K, Kadoma Y.et al. (2002) Radical scavenging activity and cytotoxicity of ferulic acid. Anticancer Res 22: 27112717.

68) Elsisi NS, Darling-Reed S, Lee EY, Oriaku ET, Soliman KF (2005) Ibuprofen and apigenin induce apoptosis and cell cycle arrest in activated microglia. Neurosci Lett 375: 91-96.

69) Hougee S, Sanders A, Faber J, Graus YM, et al. (2005) Decreased pro-inflammatory cytokine production by LPS-stimulated PBMC upon in vitro incubation with the flavonoids apigenin, luteolin or chrysin, due to selective elimination of monocytes/macrophages. Biochem Pharmacol 15; 69: 241-248.

70) Hirano T. (2004) Flavonoids such as luteolin, fisetin and apigenin are inhibitors of interleukin- 4 and interleukin- 13 production by activated human basophils. Int Arch Allergy Immunol 134:135-140.

71) Liang YC, Huang YT, Tsai SH, et.al (1999) Suppression of inducible cyclooxygenase and inducible nitric oxide synthase by apigenin and related flavonoids in mouse macrophages. Carcinogenesis 20: 1945-1952.

72) Reddy KB, Krueger JS, Kondapaka SB (1999) Mitogen activated protein kinase (MAPK) regulates the expression of progelatinase $B$ (MMP9) in breast epithelial cells. Int J Cancer 82: 268-273.

73) Nielsen SE, Young JF, Daneshvar B, et al. (1999) Effect of parsley (Petroselinum crispum) intake on urinary apigenin excretion, blood antioxidant enzymes and biomarkers for oxidative stress in human subjects. Br J Nutr Jun 81: 447-455.

74) Asghar Z, Masood Z. (2008) Evaluation of antioxidant properties of silymarin and its potential to inhibit peroxyl radicals in vitro. Pak J Pharm Sci. 21: 249-254.

75) Hiramatsu K, Tani T, Kimura Y, Izumi S, Nakane PK (1990) Effect of gamma-oryzanol on atheroma formation in hypercholesterolemic rabbits. Tokai J Exp Clin Med 15: 299-305.

76) Fahn S and the Parkinson Study Group (2005) Does levodopa slow or hasten the rate of progression of Parkinson's disease? Journal of Neurology Suppl 4:IV37-IV42. 